\title{
SOME TRAVELS IN THE LAND OF NONLINEAR CONVECTION AND MAGNETISM
}

\author{
J. Toomre ${ }^{1}$
}

\begin{abstract}
Rotating stars with convection zones are the great builders of magnetism in our universe. Seeking to understand how turbulent convection actually operates, and so too the dynamo action that it can achieve, has advanced through distinctive stages in which JeanPaul Zahn was often a central player, or joined by his former students. Some of the opening steps in dealing with the basic nonlinearity in such dynamics involved modal equations (with specified horizontal structure) to study convective amplitudes and heat transports achieved as solutions equilibrated by feeding back on the mean stratification. These dealt in turn with laboratory convection, with penetrative convection in Boussinesq settings, then with compressible penetration via anelastic equations in simple geometries, and finally with stellar penetrative convection in A-type stars that coupled two convection zones. Advances in computation power allowed 2-D fully compressible simulations, and then 3-D modeling including rotation, to revisit some of these convection and penetration settings within planar layers. With externally imposed magnetic fields threading the 2-D layers, magnetoconvection could then be studied to see how the flows concentrated the fields into complex sheets, or how new classes of traveling waves could result. The era of considering turbulent convection in rotating spherical shells had also arrived, using 3-D MHD codes such as ASH to evaluate how the solar differential rotation is achieved and maintained. Similarly the manner in which global magnetic fields could be built by dynamo action within the solar convection zone took center stage, finding that coherent wreaths of strong magnetism could be built, and also cycling solutions with field reversals. The coupling of convection and magnetism continues as a vibrant research subject. It is also clear that stars like the Sun do not give up their dynamical mysteries readily when highly turbulent systems are at play.
\end{abstract}

${ }^{1}$ JILA and Department of Astrophysical \& Planetary Sciences, University of Colorado, Boulder, CO 80309-0440, USA 


\section{Overview of our travels}

Astrophysical fluid dynamics or AFD has matured at a rapid pace in many different directions in the nearly 50 years since Jean-Paul Zahn in 1966 first published his definitive assessments of the effects of tides in the synchronization of the rotation of binary stars. As in much of theoretical astrophysics and fluid dynamics, these advances have come about because we have learned how to deal with AFD nonlinearities and far more realistic geometries and formulations. Much of this has been enabled by the remarkable improvements in computational tools and simulations. AFD has also been inspired and challenged by the refinements in observational astronomy through access to space and its own version of the digital revolution with detector systems and vast data sets. Jean-Paul Zahn and I got to first know each other in 1969 in the Woods Hole Oceanographic Institution (WHOI) yearly summer school that helped to foster and teach the theoretical tools of AFD, along with those in the closely related geophysical fluid dynamics (GFD). Edward Spiegel was quite pivotal in encouraging us and Douglas Gough to work on nonlinear treatments of thermal convection and shear flows while we were at NASA's Goddard Institute for Space Studies (GISS) in New York, and this led to what came to be called "The Convective Collective" ${ }^{1}$. Starting from those days, we have all been working some of the time on varying aspects of convection and magnetic dynamos in stellar settings, on chaotic systems, and on helioseismology and asteroseismology. We will briefly look at some examples of our mutual work with Jean-Paul. We then turn to current advances and challenges in seeking to understand the dynamics at work within our nearest star the Sun, for it can show what may also be involved in the magnetism of many other stars.

\section{Confronting nonlinear effects in convection and shear flows}

In the early 1960's, mixing-length treatments were the only means of assessing the role of turbulent convection within stellar structure and evolution models. It was unclear how nonlinearities associated with the nature of finite-amplitude motions would assert themselves, other than mixing the mean superadiabatic stratification well enough to yield a new state which would be just marginally unstable, so barely adiabatically stratified. However, the amplitude and spatial character of the resulting convective and thermal fields were quite uncertain, relying entirely on the belief that convective eddies would extend over some fraction of a vertical scale height (or thus mixing length), there to be replaced by other eddies in turn to carry the associated heat or enthalpy flux that was the central role of the convection. The early debates about the depth of the solar convection zone, such as between Öpik (1950) and Böhm-Vitense (1958) who had devised different mixing-length prescriptions for the vigor of convection, got resolved by Gough \& Weiss (1976) showing that the mixing lengths in any model had to be calibrated using the Sun

\footnotetext{
${ }^{1}$ Editor's note: see color picture in the middle of the book taken in 2004 at Newton Institute, UK.
} 
and its known luminosity, radius and age. By doing so, the depth of the solar convection settled to close its modern value deduced separately from helioseismic observations. There are also basic issues of whether the convective transport properties is mostly controlled by the local stratification, or should instead involve a radial integral of it (or thus be non-local in character), such as in overshooting of convection into adjacent regions of stable stratification (e.g., Zahn 1999).

\subsection{Modal approach to explicitly study nonlinearities in convection}

One early route to study nonlinearities in stellar convection was to greatly simplify the horizontal structure of the convection by expanding the fluctuating quantities in the Navier-Stokes equations in a set of modes which are periodic in the horizontal coordinates, and then truncating such a Galerkin expansion after a few terms. The simplest single-mode structure could involve periodic hexagonal or roll planforms, yielding nonlinear partial differential equations in the vertical coordinate and time that are parabolic in character and solvable by numerical means. The hexagons allowed study of 3-D convective motions, and the rolls in turn 2-D flows. Ed Spiegel (while passing through DAMTP in Cambridge on a sabbatical in 1967) persuaded Douglas Gough and me to join him at GISS to use such modal equations to consider nonlinear convection within the nearly incompressible Boussinesq approximation. This was appropriate for the extensive laboratory studies of turbulent convection in planar layers well under way in the 1960's.

The issue at hand was to understand what controlled the heat transport achieved by the thermal convection (measured by the Nusselt number $N$ ) as the Rayleigh number ( $R_{a}$, measure of super-criticality or nonlinearity) was varied over a large range, and how the horizontal scale (or wavenumber $a$ ) also influenced matters. This led to a gradual sequence of papers (Gough, Spiegel \& Toomre 1975; Toomre, Gough \& Spiegel 1977) that provided a mapping of $N\left(R_{a}, a\right)$ as deduced from solving the single-mode equations either numerically or by matched asymptotic expansions, with the solutions involving sensitive boundary layers that had to be resolved (Fig. 1). The resulting steady solutions could provide substantive explanations for the Nusselt number behavior with $R_{a}$ reported in the laboratory experiments, if the wavenumber $a$ was chosen to nearly maximize $N$. Although the solution of the single-mode equations could be extended to very large $R_{a}$, these still only represented highly nonlinear solutions of flows with simple cellular horizontal planforms, unlike the turbulence achieved in the experiments. Turning to more complex two- and three-mode problems (Toomre, Gough \& Spiegel 1982) yielded a medley of time-dependent solutions that made further contact with the onset of oscillatory convection seen in laboratory experiments. Although the selection of planforms and thus their modal couplings were free to be chosen, these studies emphasized the very crucial role of boundary layers in controlling the heat transport and overall convective behavior, which is indeed a hallmark of AFD. 

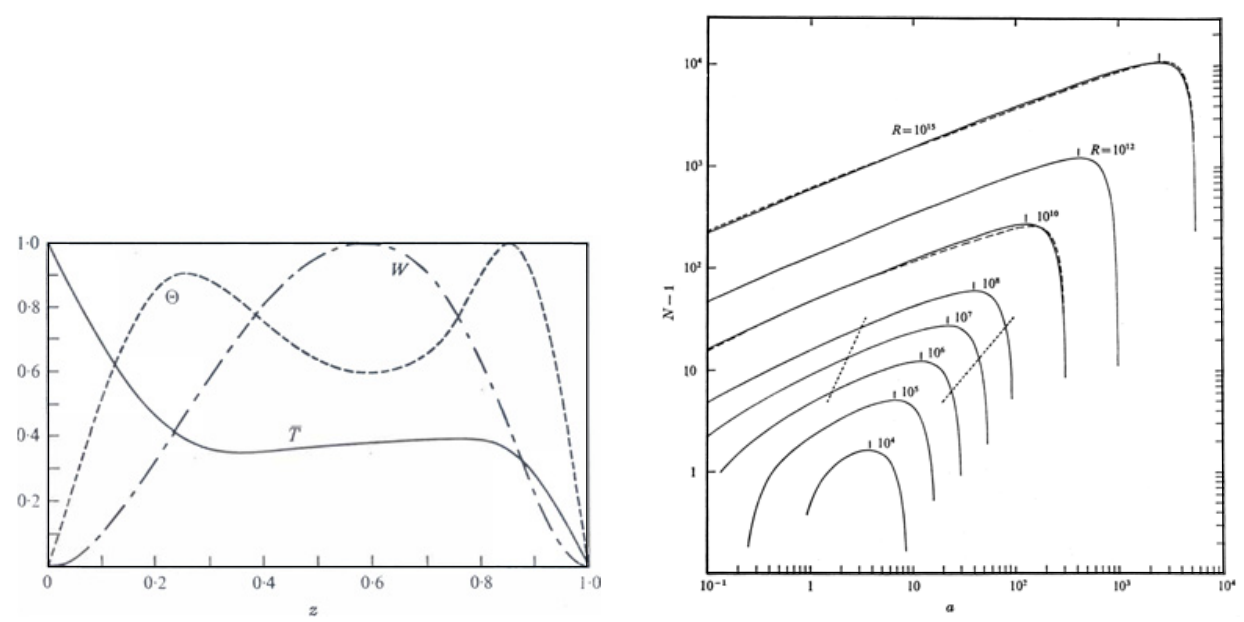

Fig. 1. Left: Variation with vertical coordinate $z$ in a typical steady single-mode hexagonal planform solution of laboratory convection at a moderate Rayleigh number $R_{a}=10^{5}$, showing the amplitude functions $W$ (vertical velocity) and $\Theta$ (fluctuating temperature), and mean temperature $\bar{T}$. Right: The heat transport as measured by Nusselt number $N$ for a wide range of selected $R$ as the wavenumber $a$ of the horizontal planforms is varied, with the solution that maximizes $N$ for given $R_{a}$ in reasonable accord with measured laboratory values. (Adapted from Gough et al. 1975; Toomre et al. 1977.)

\subsection{Finite-amplitude instability and transport in poiseuille shear flows}

Jean-Paul Zahn also came under the very positive influence of Ed Spiegel at WHOI in 1969 and tales of modal convection studies then underway. Thus he decided to join in the study of nonlinearities using modal approaches at GISS and NYU, before returning to Observatory of Nice to become its director in 1972. Jean-Paul turned to the classical puzzle of shear instabilities in Poiseuille channel flow. This topic had already received much mathematical attention and prowess in its day, and eventually required the development of matched asymptotic expansion techniques, to be able to solve the Orr-Sommerfeld equation with sufficient accuracy to show definitively that linear instability is realized when the Reynolds number $R_{e}$ exceeds about 5772. Yet laboratory experiments reported rapid transition from smooth laminar flow to turbulent spots when $R_{e}$ is greater than about 1000. Clearly some form of finite-amplitude shear instability is involved, but little progress had been made with perturbation analyses. We chose to use a Galerkin expansion with modes much as with the convection problem, though here these perturbations would be traveling with the shearing flow at phase speeds that would have to be very carefully determined along with the amplitude functions themselves in numerically solving the partial differential equations in co-moving frames. This problem turned out to be a fine adventure in obtaining both upper and lower branch solutions at different kinetic energy levels $\left(E_{U}, E_{L}\right)$ that revealed the 

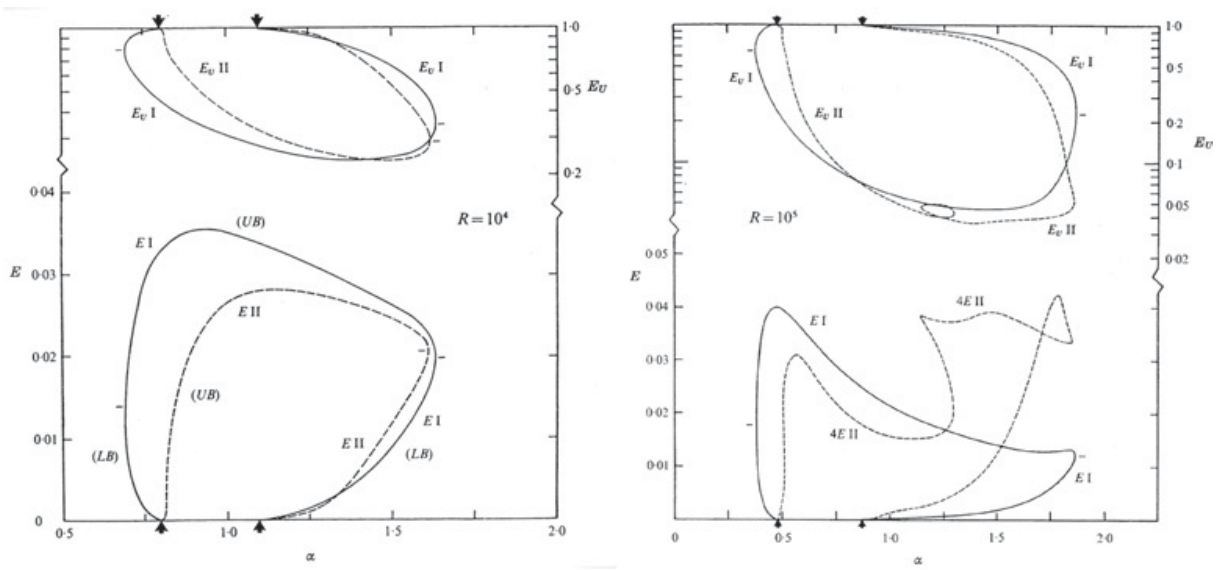

Fig. 2. Nonlinear instabilities that lead to equilibrated states as realized with one mode (I) and two-mode (II) analyses of the classic Poiseuille shear flow. Shown are kinetic energy in the fluctuating velocities $E$ and in the modified mean flow $E_{U}$ as the planform wavenumber $\alpha$ is varied at Reynolds number $R=10^{4}$ (on left) and $R=10^{5}$ (on right). The solution surfaces that emerge are remarkably complex, and possess both upper and lower branches (UB, LB) for all these travelling wave states. These finite-amplitude solutions extend to $\alpha$ values considerably greater than limits determined from linearized analyses (indicated by triangles). (Adapted from Zahn et al. 1974.)

nature of the finite amplitudes needed for shear instability (Fig. 2), and of how solutions could equilibrate to stationary states (in co-moving frames) with the mean shear profile strongly modified (Zahn, Tooomre, Spiegel \& Gough 1974). What emerged from this is that finite-amplitude perturbations involving a few modes can reduce the transition $R_{e}$ to about 2707 , and there are no runaway solutions unlike reported earlier. The mapping of the stationary solutions was a major undertaking, but clearly shear transitions from laminar to turbulent flow are much more challenging to sort out than the convective ones, and this was only a substantial first step in studying the effects of nonlinear feedbacks on instability.

\section{Nonlinear penetrative stellar convection in A-type stars}

We decided with Jean-Paul and Ed to next apply the modal convection treatment of nonlinearities to an explicit astrophysical application, namely convective transport achieved in the near-surface layers of an A-type star. We were joined at GISS by Jean Latour who had just finished his thesis work with Jean-Paul in Nice. We turned to a main sequence A star that possesses two shallow convection zones, one directly below the surface driven mainly by the ionization of $\mathrm{H}$ and another somewhat deeper down driven by the second ionization of He. These two convection zone are separated by what in most MLT models is a purely radiative 

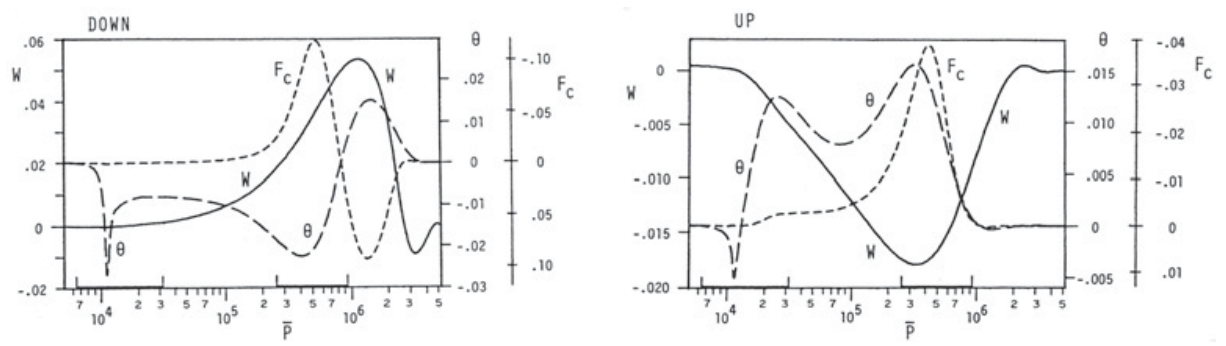

Fig. 3. Single-mode nonlinear anelastic solutions in near-surface regions of an A-type star, primarily driven within the second and deeper of the convectively unstable zones $\left(\mathrm{He}^{++}\right)$denoted by the bars in the mean pressure coordinate $\bar{p}$. Shown as variations with depth (or $\bar{p}$ ) are two solutions involving hexagonal planforms of the same horizontal wavenumber $a$, with a downward-directed hexagonal flow on the left, and an upwarddirected one on the right, displaying the vertical velocity $W$ and temperature fluctuation $\Theta$ amplitude functions and convective flux $F_{c}$. It is evident that the vigorous convection extends through the stable region between the two zones. (Adapted from Toomre et al. 1976; Latour et al. 1981.)

and quiescent zone. To carry out studies that eventually encompassed both convection zones and thus many density scale heights, we turned to the anelastic approximation that filtered out sound waves but retained other significant effects of compressibility in the modal equations (Latour et al. 1976). Our goal was to begin to probe the nature and extent of convective penetration beyond the regions of superadiabatic (unstable) stratification, and here the A star was a most convenient object with possible observable consequences. Namely, it was widely argued that the remarkable abundance of a range of metallic elements in the atmospheres of some A stars could result from radiative levitation of specific species occurring within the supposedly very quiet region between the two convection zones (e.g., Smith 1973). Although metallic-line A stars were a significant observable class, the diffusive separation of elements, which is a very slow process, could only operate in the absence of any bulk mixing.

As shown in Figure 3, our anelastic modal solutions revealed that the two convection zones are dynamically coupled by strong motions overshooting into the adjacent radiative regions (Toomre et al. 1976; Latour et al. 1981). The nonlinear single-mode equations admit two solutions for the same horizontal wavenumber $a$, with the sense of the vertical flow (up or down) at the center of a hexagonal cell influencing the extent of penetration. It emerged that convective scales comparable to solar supergranulation (as shown) would be buoyantly driven primarily in the second zone, and the flows of the upward solution would extend well into the upper convection zone. Thus there would be major mixing occurring in the intermediate radiative zone, causing real problems for the diffusive separation of elements explanations. Although these modal convection treatments are highly simplified by asserting that the flows share the same horizontal planforms over a wide range 
of depths, they were the first to begin exploring the role of nonlinearities in controlling the vigor and extent of penetration outside of stellar convection zones. To explain the origin of metallic-line A stars that are actually observed, Vauclair et al. (1978) suggested that He may gravitationally settle out sufficiently from the near-surface regions of some A stars (likely slowly rotating ones) to prevent the second convection zone from forming, thus removing our penetrative stirring mechanism and allowing diffusive separation to operate. Our modal studies served to emphasize, possibly for the first time with nonlinear tools, just how extensive can the overshooting be from a realistic stellar convection zone.

\section{Surveys of modal penetrative convection}

Our results on convection in A-type stars prompted us to step back and examine the nature of overshooting and penetration from a simpler unstable region embedded between two stable regions. By then Jean-Paul was nearly in transition from being director of Observatory of Nice to so taking over as director at Observatoire du Pic du Midi at Bagneres de Bigorre and then Toulouse. We started by surveying the nonlinear flow properties and penetration extent for one and two mode convective solutions first within an incompressible Boussinesq system. The Rayleigh number $R_{a}$ and wavenumber $a$ were varied over a wide range, and so too the degree of stiffness of the stable regions (Zahn et al. 1982). The flux of kinetic energy within 3-D hexagonal cells, whether upward or downward as determined by the sense of the central plume flow, plays a crucial role in determining the extent of penetration. Detailing scaling relations were obtained and interpreted. We sought these incompressible solutions in order to have a clear reference against which to compare penetration and its sensitivities when the effects of compressibility come seriously in play, as in many stellar settings where convection and penetration can extend over multiple density scale heights.

\subsection{Compressibility effects on penetration}

Next we turned to a comparable three-layer compressible system in which a piecewise unstable polytrope was sandwiched between two stable ones. We were joined by Josep Massaguer who was just completing his thesis work with Jean-Paul. We sought a well posed three-layer equilibrium model in which we could readily explore how the convection and its penetration were influenced by many factors, but especially by the density contrast or stratification across the unstable segment and by degree of nonlinearity as measured by $R_{a}$. This work using the anelastic modal convection equations had many surprises about asymmetries between upward and downward penetration (Massaguer et al. 1984), some features which were already present but not so recognizable in the A star studies.

Figure 4 shows two of the 3 -D single-mode hexagon solutions, one with flows directed downward at cell center and the other upward. Such solutions with a ten-fold temperature contrast across the middle unstable layer serve to emphasize the impact of compressibility. Here the penetration into the lower stable layer by 

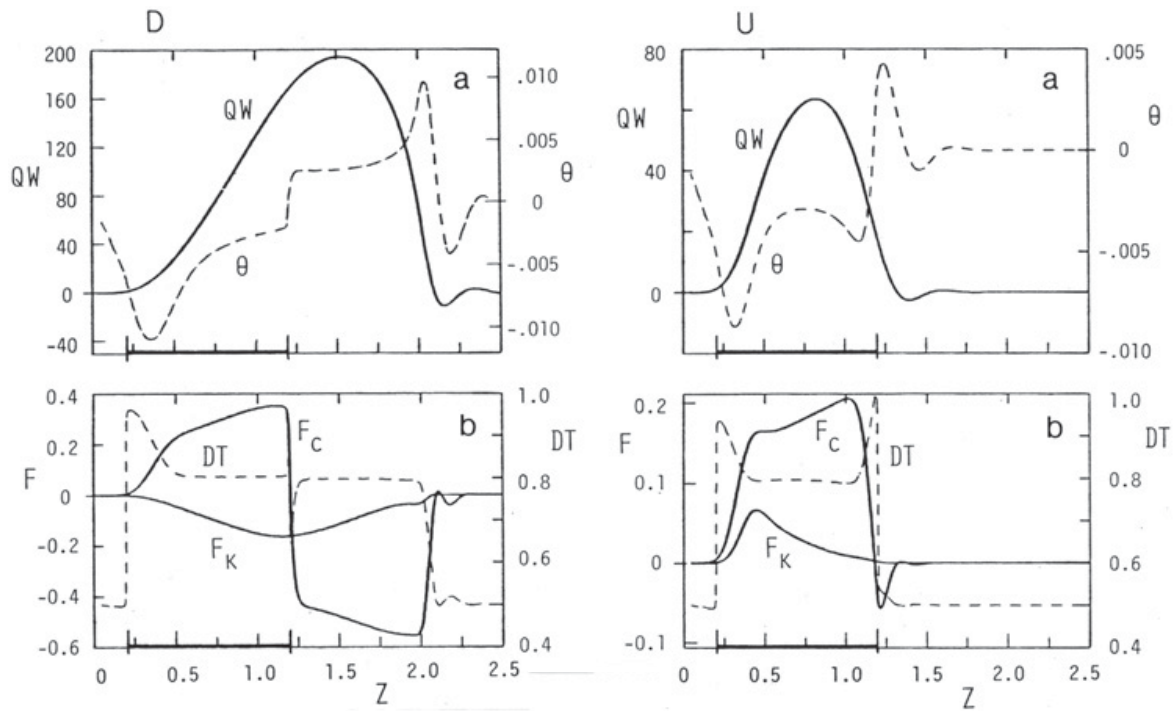

Fig. 4. Anelastic single-mode penetrative convection studied in a three-layer polytropic stratification, with the unstable middle layer denoted by the bar in depth $z$. Left: Shown in upper panel for a strongly stratified case, for the downward-directed solution, are variation with $z$ of vertical momentum $Q W$ and fluctuating temperature $\Theta$, and in lower panel the convective flux $F_{c}$ and kinetic flux $F_{k}$ and temperature gradient DT. Right: Likewise for a companion upward-directed hexagonal solution. (Adapted from Massaguer et al. 1984.)

the downward-directed plumes is considerably greater in this stratified medium than it would be in a Boussinesq system, and yields here an extended region of adiabatic stratification. In contrast, overshooting into the upper layer by the upward-directed plumes is quite modest, being sharply reduced by a remarkable reversal of the buoyancy in the upper part of the unstable layer itself if the stratification is strong. The large pressure perturbations accompanying such flows are the cause for the buoyancy reversal, these being required to drive the horizontal flows that result from deflection of the upward-directed plume flows by the shrinking vertical scale height. Thus there are intrinsic asymmetries in the mixing of stable regions above and below a stellar convection zone, which is a property realized when the density contrast of the unstable layer is large and so too the scale heights change noticeably across the whole system. The deflection of ascending flows of sufficiently wide horizontal scale, with buoyancy braking actually achieved within a convectively unstable layer itself, was a major feature that could only be revealed by such nonlinear studies. If this property carries over to more general convective flows other than these with specified horizontal planforms, it may help to explain why the largest scales of deep solar convection called 'giant cells' do not have a detectable flow signal in the surface layers where the density scale height 
has become very small (Latour et al. 1983). However, if the overall flow structures are complex, it may prove difficult to identify which scales of motion may experience buoyancy braking from others that do not.

\section{2-D Simulations of penetration below a convection zone}

Our nonlinear studies of penetrative convection were able to turn to fully compressible 2-D simulations once the computational machinery had advanced and sufficient resources became available. The modal studies had made use of the fastest supercomputers of their day, but these were not up to the task of modeling the extended evolution of convection with adequate resolution in two spatial dimensions. By about 1982 Neal Hurlburt could begin detailed 2-D convection simulations, and this initiated a new stage in our consideration with Josep Massaguer of penetration and overshooting outside an unstable zone. At about the same time, Jean-Paul in 1981 had agreed to take on the directorship of Observatoire du Pic du Midi, and to both transform this venerable institution and to join it with astronomy efforts in Toulouse. By the end of his remarkable term in 1988, the formidable Observatoire Midi-Pyrenees was emerging in Toulouse and the Pic du Midi site was modernized and financially stabilized. Jean-Paul further joined in developing a major new organization called CERFACS in Toulouse to devise new computational codes and test new supercomputing architectures in multidimension fluid simulations of importance to both science and industry. Even though Jean-Paul was very busy with all these transformative steps, we stayed in close touch through many visits in turn to both Toulouse and Boulder.

The 2-D studies began by considering compressible convection extending over multiple scale heights in a single unstable layer, revealing pronounced flow asymmetries with narrow and fast downflows and broad upflows. When the density contrast across the unstable layer was of order 10, buoyancy braking in the upflows was evident near the top due to pressure fluctuations that offset temperature fluctuations (Hurlburt et al. 1984). The next 2-D simulations considered a three-layer system of an unstable polytrope bounded above and below by stable ones, much as in the earlier modal studies (Hurlburt et al. 1986). The faster downflows led to plumes plunging into the lower layer, exciting there a broad spectrum of internal gravity waves. These waves fed back on the plumes, deflecting them sideways, and thus leading to a rich time dependence. The motions that billow upward are decidedly weaker. Jean-Paul joined us subsequently when we began to model wider 2-D domains and concentrated on just a two-layer system of highly nonlinear convection penetrating downward into stable domains with a range of relative stability parameter $S$. As shown in Figure 5, complex systems of plumes and recirculation eddies emerged, along with substantial penetration into the stable domain (Hurlburt et al. 1994). As the stiffness $S$ was increased, the pulses as evidenced in kinetic energy density $E$ were confined to narrower intervals of penetration $\nabla$. The plumes were quite effective in reasserting an adiabatic stratification over extensive portions of the stable domain, with $\nabla \propto S^{-1}$, followed by a further thermal adjustment layer. Such behavior could be interpreted by scaling 

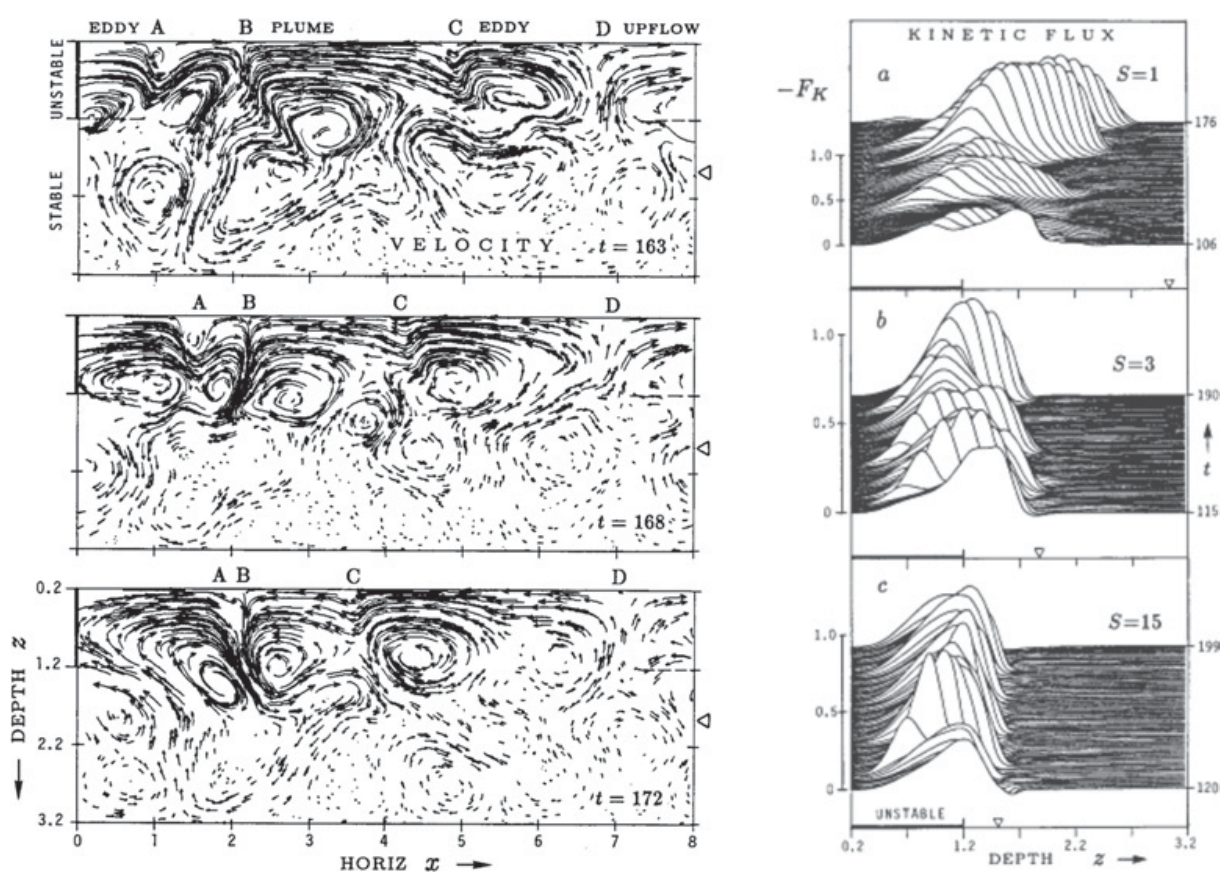

Fig. 5. Fully compressible 2-D modelling of convection in an unstable layer penetrating into a stable domain below. Left: Velocity field streaklines in three successive snapshots of vigorous convection for $S=3$ involving strong downward-directed plumes being swung laterally by the gravity wave field they initiate. Right: Kinetic energy density $E$ as function of time and depth $z$ for three simulations with increasing relative stiffness $S$ of the stable domain, with extent of effective penetration (denoted by triangle) sharply reduced with increasing $S$. (Adapted from Hurlburt et al. 1994.)

arguments that Jean-Paul devised. These 2-D studies served to provide a broad set of estimates of the effectiveness of compressible convection to stir and mix the surrounding stable layers without resorting to rather uncertain MLT arguments. Further advances in computing allowed 3-D modeling of similar configurations in due course. These showed that the filling factor of downward-directed plumes was considerably lesser in 3-D, and although the penetration and mixing extent $\nabla$ was comparable, the flows were far less effective in establishing an extended adiabatic stratification there (e.g., Brummell et al. 2002). Thus the recent 3-D studies suggest that overshooting of motions is roughly in accord with the 2-D models, but an extended abiabatic region is not.

\section{Solar internal dynamics and surface magnetism}

Let us now turn to our more recent travels in the land of convection and magnetism, a subject in which Jean-Paul was consistently very interested though could 
not devote much of his attention. That was increasingly required for the graduate school in astrophysics at Observatoire de Paris-Meudon that he started to coordinate and run for five years starting in 1995 after returning to Paris in 1993. Many of our modern travels with nonlinear convection and magnetism are closely tied to our nearest star the Sun, for it provides a remarkable level of observational detail not feasible with any other star to both challenge and guide theoretical efforts. We hope that our Sun is also representative in the larger aspects of magnetism shared by many stars in our universe. So let us very briefly review some of the salient solar observational features, and then turn to a sampling of recent results from convection and dynamo theory that suggest some of the paths that may be pursued in the next few years.

The Sun as a magnetic star exhibits a wide variety of activity, ranging from the fairly regular 11-year cycles of major sunspot eruptions with orderly patterns on the global scales to the intense and more chaotic magnetic fields on the small scales. The strong magnetic fields that emerge through the solar surface are pivotal in controlling the evolving structure of the Sun's chromosphere and extended corona. Such complex magnetism presents major challenges to heliophysics theory as we seek to understand how the Sun, like many other stars, builds the intense large-scale magnetic fields so evident at its surface. Solar magnetic activity also acutely impacts our technological society, thus providing a strong impetus to understand its origins. During the peak phases of the Sun's 11-year magnetic cycle, particles and radiation from coronal mass ejections and explosive flares can damage satellites, endanger astronauts, disrupt power grids, divert air flights, and interrupt telecommunications. Such events are clearly linked to the evolving magnetic fields within the extended solar atmosphere and heliosphere, and these are largely driven by the emergence of magnetic flux from the solar interior on a wide range of spatial scales. The origin of these solar magnetic fields must ultimately rest with dynamo processes occurring in the solar interior.

\subsection{Helioseismic probing of solar interior flows}

Helioseismology permits us to probe and assess flows deep within the Sun in ever increasing detail (Fig. 6). It provides important guidance and constraints to dynamo theory in terms of the differential rotation profile, meridional circulation patterns, and the large-scale subsurface flows interacting with active regions (e.g., Howe 2009; Charbonneau, 2010). Indeed, helioseismic probing has revealed that two boundary layers are present, with both the near-surface shear layer and the tachocline at the base of the convection zone $(\mathrm{CZ})$ being sites of enhanced rotational shear that can participate in dynamo action. The interior dynamics continues to be probed with greater fidelity with the Helioseismic and Magnetic Imager (HMI) on the Solar Dynamics Observatory (SDO), as it is now providing continuous high-resolution measurements of Doppler velocity and vector magnetic fields across the full solar disk. The Atmospheric Imaging Assembly (AIA) complements HMI by revealing what is occurring simultaneously in magnetic structures over global scales and a wide range of heights in the solar atmosphere. We are 

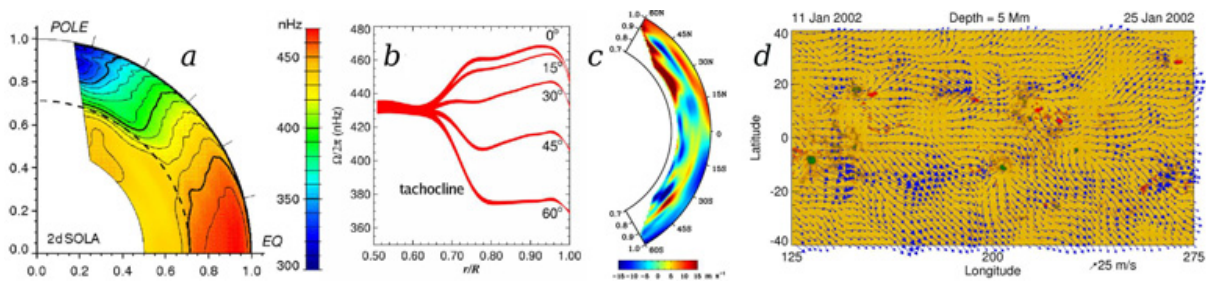

Fig. 6. (a) Angular velocity profile $\Omega$ from helioseismology showing differential rotation within the solar interior, with fast rotation (red) at the equator and slow (blue) at poles. (b) Radial cuts of $\Omega$ at different latitudes. The prominent boundary layers of the tachocline at $0.71 R_{\odot}$ and near-surface shear layer in the outer $5 \%$ by radius are evident (Thompson et al. 2003). (c) Meridional flows with depth and latitude as recently determined with time-distance methods, suggesting multi-cell structures within a hemisphere (Zhao et al. 2013). (d) Synoptic map of horizontal flows of solar subsurface weather (SSW) at $5 \mathrm{Mm}$ depth, with surface magnetic field overlayed (Featherstone 2011; Greer et al. 2015).

carrying out helioseismic probing of subsurface flows with our new high-resolution ring diagram techniques (Greer et al. 2015; Greer et al. 2016a, 2016b) applied to HMI Doppler data. We are also very actively using the HMI and MDI magnetic imaging data to complement interpretation of these helioseismic flow analyses.

\subsection{Nature and modeling of the tachocline}

The tachocline had not been anticipated prior to its discovery by helioseismology. This boundary layer of strong rotational shear near the base of the CZ $(r \sim$ $\left.0.71 R_{\odot}\right)$ is a complex transition layer between the prominent differential rotation above and the uniform rotation of the deep radiative zone (RZ) below. Although the tachocline appears in Figure $6(\mathrm{~b})$ to be about $0.05 R_{\odot}$ in thickness, that likely reflects just the resolution of the helioseismic inversion at that depth, and the tachocline may well be much narrower. It has been variously proposed that the rotational shear of the tachocline is confined by effects of anisotropic turbulence (Spiegel \& Zahn 1992), by gravity waves (e.g., Talon, Kumar \& Zahn 2002), or by magnetic stresses (e.g., Gough \& MacIntyre 1998). Indeed, Jean-Paul and Ed were the first to provide a possible explanation for the existence of the tachocline. Turbulent and laminar stresses associated with convective overshoot, magnetic pumping, instabilities and breaking waves can also influence the structure of the tachocline and the transport of angular momentum across it. How and why such a thin rotational boundary layer has arisen in the Sun is still an open research topic, as reviewed by Miesch (2005) and Miesch \& Toomre (2009), and discussed here by Douglas Gough ${ }^{2}$. There is much interest in the role that the presence of

\footnotetext{
${ }^{2}$ Editor's note: see D. Gough's contribution these proceedings.
} 
a tachocline has on the operation of magnetic cycles, and on the storage, destabilization, and buoyant rise of coherent toroidal fields. All these are crucial elements when seeking to understand flux emergence that yields the sunspots.

There is growing experience with global simulations in incorporating a region of stable stratification at the base of the $\mathrm{CZ}$ into which plumes can overshoot, thereby modeling the transition into the radiative zone (e.g., Browning et al. 2006; Ghizaru et al. 2010; Racine et al. 2011). The prominent differential rotation with latitude so evident in the $\mathrm{CZ}$ can be prevented from diffusively spreading into the uniformly rotating RZ by sharply reducing the SGS diffusivities in the quiescent layer below the active overshooting, mimicking the expected decrease in the intensity of smallscale turbulence (Brun et al. 2011; Strugarek et al. 2011; Augustson et al. 2013; Alvan et al. 2014). Care must be taken with the subtleties of energy conservation in this region (Brown et al. 2012; Vasil et al. 2013).

\subsection{Sunspot emergence calling cards}

The Sun shows that magnetism in stars involves both beauty and complexity. The large-scale fields seen as sunspots and active regions follow fairly orderly and systematic 11-year cyclic patterns that have long been studied (e.g., Stix 2002; Charbonneau 2010; Hathaway 2010). Joining these are small-scale magnetic fields that appear to be nearly random in time and space, often referred to as a "salt and pepper' pattern or the "magnetic carpet" as viewed with TRACE and now with HMI and AIA on SDO (e.g., Schrijver et al. 1997, 2013; Stenflo \& Kosovichev 2012). Both the large-scale and small-scale components contribute to the total magnetic activity of the Sun. A clear challenge is to understand how solar dynamo action within the interior can yield such diverse and evolving magnetic fields.

The "butterfly diagram" of sunspot emergence with latitude and time as the solar cycles advance is the striking hallmark of solar variability. When accompanied with plots in time of the total area occupied by sunspots which wax and wane over the course of about 11 years, these two figures exemplify the prominent character of the magnetic Sun (Figs. 7a,b). These are the two "calling cards" of solar physics. Aspects of cycling were first recognized by Schwabe in 1843, and became the goal of daily sunspot observations as various astronomical observatories were established. Despite such prominence, there is still little theoretical understanding of why the sunspots emerge as they do. The sunspot behavior is the primary challenge to all basic solar dynamo theory.

The advent of space-based assets to provide nearly continuous full-disk observations, such as first with SOHO and now with SDO, accompanied with data from the ground-based GONG network, has greatly improved the fidelity of these records of sunspot and active region evolution as the cycles proceed. This has enabled radial magnetic field mappings averaged in longitude over successive solar rotations, as in Figure 7c, with the butterfly signature of sunspots clearly visible at lower latitudes, along with fainter streaks of new polarity extending to the poles. More importantly, space has given us access to the UV and X-ray portions of the spectrum that reveal the complex loops and arcs and CMEs of evolving magnetism 


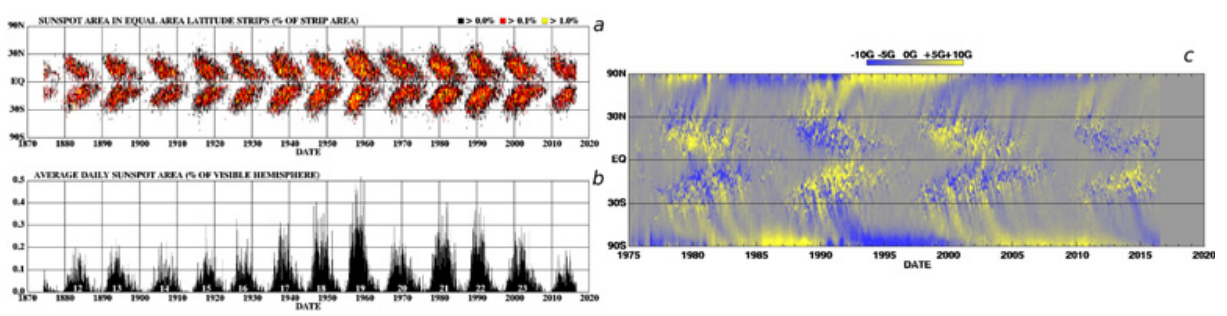

Fig. 7. Solar variability and sunspot butterfly diagrams. (a) Detailed observations of sunspots obtained by RGO since 1874, and updated with modern data. These show that emerging sunspots are concentrated in two latitude bands on either side of equator, forming first at mid latitudes, widen, and then move toward the equator as each cycle progresses. (b) The cycle is at a minimum as the sunspot emergence nears the equator, at which time new spots are also beginning to form at mid-latitudes. (c) Modern mapping of evolving radial magnetic field associated with sunspots and active regions, averaged in longitude over successive solar rotations, showing polarity of field, based on MDI and HMI observations. (Hathaway 2016.)

in the chromosphere and corona that accompany flux emerge in the photosphere. The web has transformed access to such superb and beautiful data, as exemplified by both AIA and HMI magnetic and Doppler imaging at $4096^{2}$ resolution available shortly after their acquisition by SDO, as Alan Title has discussed. The sunspot flux emergence is the primary metronome that drives much of the rich behavior in the solar atmosphere, and thus its origin deserves renewed attention.

\subsection{Elements of global dynamo}

The observed solar magnetic field exists over a vast range of spatial scales, from the global-scale coronal structures to the chaotic smallest scales of the rapidly evolving photospheric fields. These fields are thought to be generated by two linked but conceptually distinct dynamos. A local small-scale dynamo generates the smallest magnetic structures seen on the photosphere amidst the intense turbulence of the near-surface layers of the Sun. In contrast, a global-scale dynamo operating within the depths of the $\mathrm{CZ}$ and tachocline builds the large-scale fields observed in the solar atmosphere. Indeed, global-scale convective dynamo simulations show that two mechanisms dominate the generation of both large-scale toroidal and poloidal fields. Within the context of mean-field theory (MFT), these are the $\alpha$-effect and the $\Omega$-effect. The $\alpha$-effect encapsulates the net action of small-scale helical flows, which are inherently present within turbulent rotating convective systems, on axisymmetric toroidal fields that produces poloidal fields. These systems also self-consistently generate a differential rotation, which leads to the $\Omega$-effect through the stretching and shearing of axisymmetric poloidal magnetic field into toroidal field. 
Despite the dominance of these basic mechanisms in the dynamo operating within the bulk of the $\mathrm{CZ}$, the impact of a stably stratified tachocline upon them is still largely unknown. Some ideas are provided by the concept of an interface dynamo (e.g., Parker 1993), which provides one intuitive picture of how the global dynamo may be modified in the presence of a tachocline (e.g., Ossendrijver 2003; Fan 2009; Charbonneau 2010, 2014; Miesch 2012). In this picture, the poloidal field generated by cyclonic turbulence within the bulk of the CZ, or by breakup of active regions, is pumped downward to the tachocline ( $\gamma$-effect in MFT). The radial differential rotation provided by the tachocline can then stretch that field into strong toroidal structures. Portions of those structures may succumb to magnetic buoyancy instabilities and rise upwards to pierce the photosphere as looped structures that form the observed active regions. These distinct processes can now be resolved in modern 3-D convective dynamo simulations. It is within the context of these potential mechanisms that we should study the influence of a tachocline upon an actual 3-D global-scale convective dynamo.

The emergence of magnetic flux in the form of active regions not only powers solar variability, but it may also play a central role in the operation of the dynamo itself. This is the premise of Babcock-Leighton/Flux-Transport (BL/FT) dynamo models in which the buoyant destabilization, emergence, and dispersal of magnetic flux structures generates a mean poloidal field that helps to sustain magnetic cycles and the meridional circulation plays a key role in setting the cycle period (Dikpati \& Gilman 2006; Rempel 2006; Munoz-Jaramillo et al. 2010; Pipin \& Kosovichev 2013; Charbonneau 2010, 2014). Reconciling this BL/FT kinematic model with a dynamically self-consistent turbulent convective global dynamo requires a better understanding of how and where these emergent flux structures are actually formed.

\section{Recent advances in global dynamo modeling with 3-D simulations}

Studying elements involved in the solar dynamo is made challenging by the vast range of length and time scales involved in the highly nonlinear dynamics of the deep convection zone (CZ, occupying the outer $29 \%$ or $200 \mathrm{Mm}$ by radius below the surface). These scales extend from the intense small-scale turbulence of granulation and supergranulation visible in the surface layers to the global-scale deep flows of giant cells, differential rotation and meridional circulation. The theoretical efforts must thus deal with stratified and rotating magnetohydrodynamic (MHD) turbulence in fully 3-D settings. Many complex dynamical elements are involved, including the differential rotation of the $\mathrm{CZ}$ and the tachocline of shear at its base, turbulent production and transport of the magnetic fields by the convection, shear amplification of the fields, and magnetic buoyancy that leads to the eventual field eruption. In ongoing studies of the solar dynamo, major progress has been achieved with modeling the coupling of turbulent convection, shear and magnetism in rotating spherical shells of conducting fluids, representative of the solar CZ and its tachocline. Using 3-D global hydrodynamic simulations (Fig. 8a,b) with our Anelastic Spherical Harmonic (ASH) code, our studies have achieved solar-like 

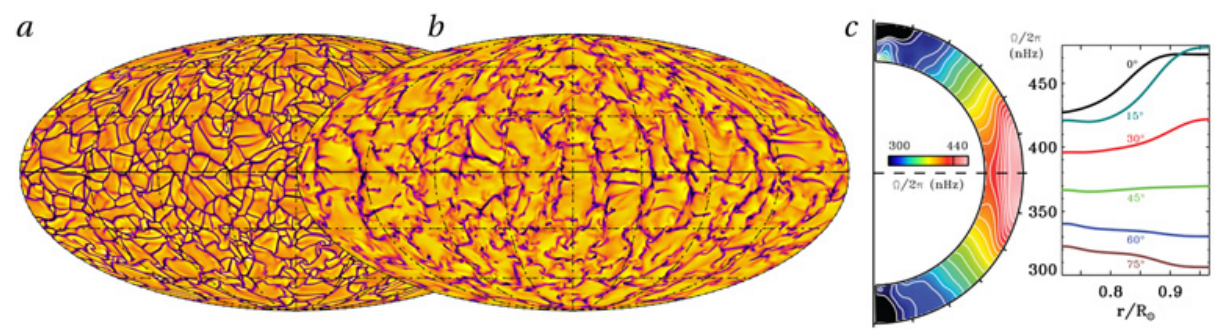

Fig. 8. High-resolution global solar convection ASH simulation (Miesch et al. 2008), showing the connectivity of the major downflows with depth as the giant cells become more evident. Snapshot of radial velocity $v_{r}$ in Mollweide projection (downflows are dark) at $(a) 0.98 R_{\odot}$ near the top of the domain and $(b)$ at mid-convection zone $\left(0.92 R_{\odot}\right)$ detailing the largest scale flows. Cyclonic swirl is evident in many of the downflow interstices. Emergence of new downflow lanes leads to continual restructuring of the giant convection cells. (c) Separate ASH modeling with a specified thermal structure in the tachocline (Miesch et al. 2006) yields a solar-like mean angular velocity $\Omega$ profile with radius and latitude (along with radial cuts of $\Omega$ at indicated latitudes) which make contact with helioseismic deductions.
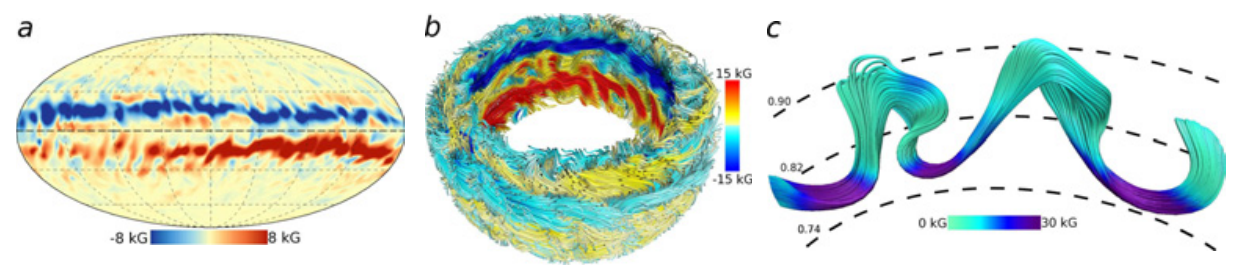

Fig. 9. Global stellar dynamo simulations with $\mathrm{ASH}$ of turbulent MHD convection in a younger Sun rotating at three times the current solar rate (case S3; 8 day period). (a) Prominent wreaths of strong toroidal magnetic fields are realized, with opposite polarity above and below the equator in a global Mollweide mapping. (b) Volume rendering of magnetic field lines shows the complex structure within these persistent wreaths (Nelson et al. 2013). (c) Buoyant loop segment rising from a magnetic wreath, colored by field strength (Nelson et al. 2011, 2014).

differential rotation profiles (Fig. 8c) that are largely in accord with helioseismic findings (Fig. 6a,b).

\subsection{Magnetic wreath building dynamos}

Turning to global MHD simulations with ASH, our simulations have revealed that remarkable wreaths of strong magnetic field (Fig. 9) can be built in the bulk of the $\mathrm{CZ}$ by dynamo action. These wreaths of toroidal magnetic fields often have opposite polarity in the two hemispheres. The sense of the magnetic fields can 

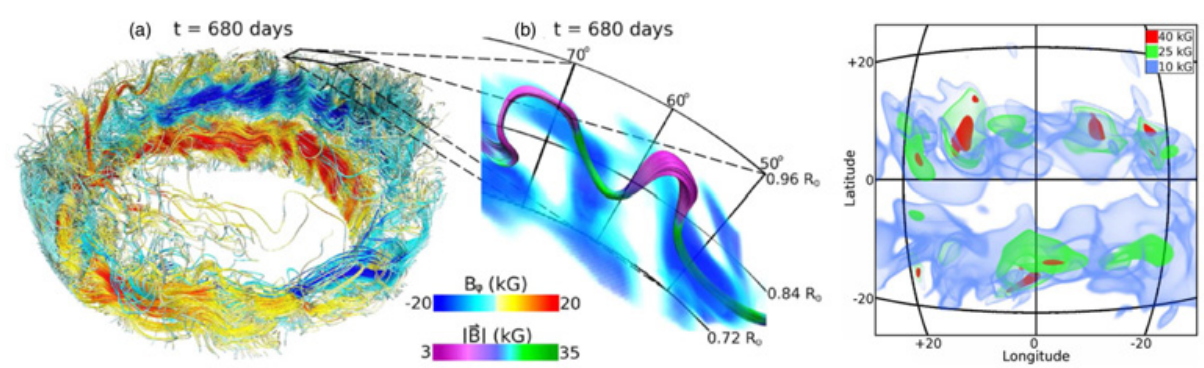

Fig. 10. Global stellar dynamo simulations with ASH of turbulent MHD convection in a younger Sun rotating at three times the current solar rate (case S3; 8 day period). (a) Prominent wreaths of strong toroidal magnetic fields are realized, with opposite polarity above and below the equator. (b) Buoyant loops of magnetism are self-consistently achieved and transit the convection zone. (c) The buoyant loops form in the strongest regions of magnetic field, shown here in volume rendering (Nelson et al. 2013).

reverse in a cyclic manner over decade-long time scales. One of our most recent ASH simulations (case K3S) passed through many reversing cycles (Fig. 11) before experiencing brief quiescent intervals much like a Maunder Minimum. Interestingly, whereas the primary cycling at low latitudes was interrupted, the cycling at high latitudes continued, but without reversals. After the hiatus, the model resumed its regular cycling. These processes involve sensitive balances between magnetic field amplification and dissipation that occur over a range of scales, with multi-scale interactions being critical to the field evolution.

Another step forward with ASH has been to attain the first global convective dynamo simulation (case S3) to exhibit the spontaneous, self-consistent generation of rising magnetic loops, as shown in Figures 9c, 10. Over 130 such loops have been identified. By "self-consistent" we mean that convection under the influence of rotation generates a differential rotation that in turn generates a coherent toroidal field that in turn destabilizes and rises upward through the $\mathrm{CZ}$. The rise is due to a combination of magnetic buoyancy and advection by the convective motions, which work together to move flux upward. Figure 10 illustrates the topology of the field both globally and locally in a buoyant loop. The distribution of loop tilts near the top of their ascent makes contact with Joy's Law.

It is remarkable that neither case K3S nor case S3 has a tachocline. This is telling us that a tachocline is not crucial to achieve cyclic magnetic activity or for flux emergence in the form of bipolar magnetic regions. On the other hand, other global simulations with codes including ASH are showing that the presence of a tachocline can promote the generation and storage of strong toroidal magnetic fields (Browning et al. 2006; Racine et al. 2011; Masada et al. 2013; Augustson et al. 2013) and longer magnetic cycles (Racine et al. 2011; Guerrero et al. 2015). Yet none of those have led to self-consistent loop generation from the tachocline. This is an aspect deserving of close attention. 

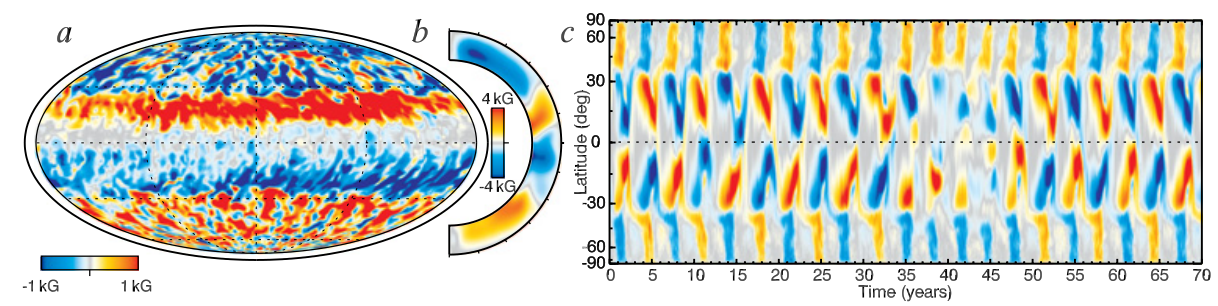

Fig. 11. Nature of toroidal magnetic field $B_{\phi}$ in an ASH dynamo simulation with slopelimited diffusion (SLD, case K3S). (a) Snapshot of horizontal structure of $B_{\phi}$ in a global view (Mollweide projection) at $0.95 R_{\odot}$, showing the connectivity of the magnetic wreaths (field polarity in color). (b) Longitude-averaged $\left\langle B_{\phi}\right\rangle$, also averaged in time over an energy cycle, shown in a meridional plane, revealing that wreaths at lower latitudes occupy much of the convection zone depth. (c) Time-latitude diagram of $\left\langle B_{\phi}\right\rangle$ at $0.95 R_{\odot}$, exhibiting repeated cycles of equatorward migration of the wreaths (from the tangent cylinder at latitude $40^{\circ}$ ), and the poleward propagation of the field at higher latitudes. The cycling enters a grand minimum, after which reversing cycling resumes. (Augustson 2013; Augustson et al. 2014, 2015.)

In overview, the last seven years have seen dramatic progress in 3-D modeling of the cyclic solar dynamo, using several different codes including ASH now capable of studying at least portions of rotating spherical shells. These have revealed unprecedented magnetic self-organization, with strong mean-field generation in highly nonlinear dynamo regimes involving wreaths (Brown et al. 2010). There is also cyclic activity on decadal time scales (Ghizaru et al. 2010; Brown et al. 2011a, 2011b; Racine et al. 2011; Nelson et al. 2013; Fan \& Fang 2014, 2015; Hotta et al. 2016). And we are seeing equatorward propagation of toroidal flux in cycling systems (Augustson et al. 2015; Käpylä et al. 2013), as shown in Figure 11(c). However, the detailed influence of a tachocline on this latest generation of solar global convective dynamos has just begun to be explored.

\subsection{Near-surface convection and dynamo action}

There are also recent striking results from 3-D radiative MHD modeling of nearsurface solar convection and dynamo action carried out in localized domains. Some study quiet Sun magnetism from the small-scale dynamo (e.g., Rempel 2014); others follow the rise of an introduced semi-torus of magnetic field and study how its ascent through the granular and larger convection leads to active regions (e.g., Cheung et al. 2010; Rempel \& Cheung 2014); and others consider how uniform horizontal magnetic field introduced at the base of this shallow layer (but deep in scale heights) can be reorganized by the turbulent convection into a hierarchy of compact looped structures that break through the surface, the largest yielding a small active region with two spots (e.g., Stein et al. 2011; Stein \& Nordlund 2012). Bipolar magnetic regions are also realized in a stratified two-layer system with 
forced turbulence in which a weak imposed horizontal field is strongly amplified (e.g., Warnecke et al. 2016). These studies can illuminate how actual sunspots may emerge from the broader ascending loops of magnetic field obtained in our global simulations that cannot yet get all the way to the surface. They also serve to explore whether near-surface convection by itself can form the smaller sunspots. Our global approach with ASH is complementary by providing a Hale-like law of hemispherical polarity reversal for the emerging structures that can be further concentrated when they pierce through the surface.

\section{Final reflections}

Astrophysical fluid dynamics is a vibrant subject in very many areas, and clearly so in trying to understand how stars build their magnetic fields through the complex coupling of convection, rotation and shear within an electrically conducting fluid. As we have seen with the theoretical efforts underway in solar physics which are both guided and challenged by observations, work in dealing with highly nonlinear convection and magnetism is making prominent advances. Jean-Paul placed great emphasis on clarifying basic elements in the dynamics that would carry over to many astrophysical problems, including those that contribute to dynamo action within stars. Although he did not work much on MHD processes himself, he taught and inspired young colleagues who now are leading many aspects of research on solar and stellar magnetism. The intense turbulence present within most of these real problems is certainly daunting. Yet it appears that we can make some real headway toward understanding the operation of dynamos through 3-D numerical simulations that capture many of the basic nonlinear elements. The continuing rapid advances in computation are allowing us to carry out, what are in essence, ever better numerical gedanken experiments bearing on stellar magnetism. It is remarkable that many of the features emerging from these latest $3-\mathrm{D}$ simulations are making contact with observed solar cycle properties, even though being far from capturing the vast dynamical ranges inherent in the real physical systems. We do not quite know why, nor can we defend all our choices in simplifying the physics. Being optimists, or at least hopeful travelers, we see an interesting road ahead in these journeys through the joys of nonlinear dynamics.

These travels through the land of convection and magnetism have involved joint work with many of our fellow participants in this celebration of the life and scientific creativity of Jean-Paul Zahn. I am here the messenger of some of what has occurred. For the more recent collaborations, I am especially indebted to Kyle Augustson, Benjamin Brown, Matthew Browning, Sacha Brun, Nicholas Featherstone, Douglas Gough, Benjamin Greer, Bradley Hindman, Mark Miesch and Nicholas Nelson. NASA has provided partial support through grants NNX11AJ36G, NNX13AG18G and NNX14AB56G. 


\section{References}

Alvan, L., Brun, A.S. \& Mathis, S., 2014, Astron. \& Astrophys., 565, A42

Augustson, K.C., 2013, "Convection and Dynamo Action in Massive Stars", Ph.D. Thesis (University of Colorado), p. 430

Augustson, K.C., Brun, A.S., \& Toomre, J., 2013, Astrophys. J., 777, 153

Augustson, K.C., Brun, A.S., Miesch, M.S., \& Toomre, J., 2015, Astrophys. J., 809, 149

Böhm-Vitense, E., 1958, Zs. f. Astrophys., 46, 108

Brown, B.P., Browning, M.K., Brun, A.S., Miesch, M.S., \& Toomre, J., 2010, Astrophys. J., 711,424

Brown, B.P., Browning, M.K., Brun, A.S., Miesch, M.S., \& Toomre, J., 2011a, Astrophys. J., 731,69

Brown, B.P., Browning, M.K., Brun, A.S., Miesch, M.S., \& Toomre, J., 2011b, in Proc.16th Cool Stars, ASPCS, 448, 277

Brown, B.P., Vasil, G.M., \& Zweibel, E.G., 2012, Astrophys. J., 756, 109

Browning, M.K., Brun, A.S., Miesch, M.S., \& Toomre, J., 2006, Astrophys. J.L, 648, L157

Brummell, N.H., Clune, T.L., \& Toomre, J., 2002, Astrophys. J., 570, 825

Brun, A.S., Miesch, M.S., \& Toomre, J., 2004, Astrophys. J., 614, 1073

Brun, A.S., Miesch, M.S., \& Toomre, J., 2011, Astrophys. J., 742, 79

Charbonneau, P., 2010, Living Rev. Solar Phys., 7, 3

Charbonneau, P., 2014, Ann. Rev. Astron. Astrophys., 52, 251

Cheung, M.C.M., Rempel, M., Title, A.M., \& Schussler, M., 2010, Astrophys. J., 720, 233

Dikpati, M., \& Gilman, P.A., 2006, Astrophys. J., 649, 498

Fan, Y., 2009, Living Rev. Solar Phys., 6, 4

Fan, Y., \& Fang, F., 2014, Astrophys. J., 789, 35

Featherstone, N.A., 2011, "Exploring Convection and Dynamos in the Cores and Envelopes of Stars", Ph.D. Thesis (University of Colorado), p. 174

Ghizaru, M., Charbonneau, P., \& Smolarkiewicz, P., 2010, Astrophys. J. Lett., 715, L133

Gough, D.O., Spiegel, E.A., \& Toomre, J., 1975, J. Fluid Mech., 68, 696

Greer, B., Hindman, B.W., Featherstone, N.A., \& Toomre, J., 2015, Astrophys. J. Lett., 803, L17

Greer, B., Hindman, B.W., \& Toomre, J., 2016a, Astrophys. J., 824, 4

Greer, B., Hindman, B.W., \& Toomre, J., 2016b, Astrophys. J., 824, 128

Gough, D.O., \& McIntyre, M.E., 1998, Nature, 394, 755

Gough, D.O., \& Weiss, N.O., 1976, Mon. Not. Roy. Astr. Soc., 176, 589

Guerrero, G., Smolarkiewicz, P.K., de Gouveia Dal Pino, E.M., Kosovichev, A.G., \& Mansour, N.N, 2015, Astrophys. J., 819, 104

Hathaway, D.H., 2010, Living Rev. Solar Phys., 7, 1

Hathaway, D.H., 2016, http://solarscience.msfc.nasa.gov/SunspotCycle.shtml, regularly updated by Hathaway

Hotta, H., Rempel, M., \& Yokoyama, T., 2016, Science, 351, 1427

Howe, R., 2009, Living Rev. Solar Phys., 6, 1 
Hurlburt, N.E., Toomre, J., \& Massaguer, J.M., 1984, Astrophys. J., 282, 557

Hurlburt, N.E., Toomre, J., \& Massaguer, J.M., 1986, Astrophys. J., 311, 563

Hurlburt, N.E., Toomre, J., Massaguer, J.M., \& Zahn, J.-P., 1994, Astrophys. J., 421, 245

Käpylä, P.J., Mantere, M. J., Cole, E., Warnecke, J., \& Brandenburg, A., 2013, Astrophys. J., 778, 41

Latour, J., Spiegel, E.A., Toomre, J., \& Zahn, J.-P., 1976, Astrophys. J., 207, 233

Latour, J., Toomre, J., \& Zahn, J.-P., 1981, Astrophys. J., 248, 1081

Latour, J., Toomre, J., \& Zahn, J.-P., 1983, Solar Phys., 82, 387

Masada, Y., Yamada, K., \& Kageyama, A., 2013, Astrophys. J., 778, 11

Massaguer, J.M., Latour, J., Toomre, J., \& Zahn, J.-P., 1984, Astron. \& Astrophys., 140,1

Miesch, M.S., 2005, Living Rev. Solar Phys., 2, 1

Miesch, M.S., 2012, Phil. Trans. Roy. Soc. A, 370, 1970, 3049

Miesch, M.S., \& Brown, B.P., 2012, Astrophys. J. Lett., 746, L26

Miesch, M.S., Brun, A.S., \& Toomre, J., 2006, Astrophys. J., 641, 618

Miesch, M.S., Brun, A.S., DeRosa, M.L., \& Toomre, J., 2008, Astrophys. J., 673, 556

Miesch, M.S., \& Toomre, J., 2009, Ann. Rev. Fluid Mech., 41, 317, and on-line appendix, "Tachocline confinement" (6pp)

Munoz-Jaramillo, A., Nandy, D., Martens, P.C.H., \& Yeates, A.R., 2010, Astrophys. J. Lett., 720, L20

Nelson, N.J., 2013, "Magnetic Wreaths, Cycles and Buoyant Loops in Convective Dynamos", Ph.D. Thesis (University of Colorado), p. 220

Nelson, N.J., Brown, B.P., Brun, A.S., Miesch, M.S., \& Toomre, J., 2011, Astrophys. J. Lett., 739, L38

Nelson, N.J., Brown, B.P., Brun, A.S., Miesch, M.S., \& Toomre, J., 2013, Astrophys. J., 762,73

Nelson, N.J., Brown, B.P., Brun, A.S., Miesch, M.S., \& Toomre, J., 2014, Solar Phys., 289,441

Öpik, E.J., 1950, Mon. Not. Roy. Astr. Soc., 110, 559

Ossendrijver, M., 2003, Astron. Astrophys. Rev., 11, 287

Parker, E.N., 1993, Astrophys. J., 408, 707

Pipin, V.V. \& Kosovichev, A.G., 2013, Astrophys. J., 776, 36

Racine, E., Charbonneau, P., Ghizaru, M., Bouchat, A., \& Smolarkiewicz, P., 2011, Astrophys. J., 735, 46

Rempel, M., 2006, Astrophys. J., 647, 662

Rempel, M., 2014, Astrophys. J., 789, 132

Rempel, M., \& Cheung, M.C.M., 2014, Astrophys. J., 785, 90

Schrijver, C.J., Title, A.M., Van Ballegooigen, A.A., Hagenaar, H.G., \& Shine, R.A., 1997, Astrophys. J., 487, 424

Schrijver, C.J., Title, A.M., Yeats, A.R. \& DeRosa, M.L., 2013, Astrophys. J., 773, 93

Smith, M.A., 1973, Astrophys. J.S, 25, 277

Spiegel, E.A., \& Zahn, J.-P., 1992, Astron. \& Astrophys., 265, 106

Stein, R.F., Lagerfjard, A., Nordlund, A., \& Georgobiani, D., 2011, Solar Phys., 268, 271 
Stein, R.F., \& Nordlund, A., 2012, Astrophys. J., 753, L13

Stenflo, J.O., \& Kosovichev, A.G., 2012, Astrophys. J., 745, 129

Stix, M., 2002, The Sun (Springer, New York)

Strugarek, A., Brun, A.S., \& Zahn, 2011, Astron. \& Astrophys., 532, A34

Talon, S., Kumar, P., \& Zahn, J.-P., 2002, Astrophys. J., 574, L175

Thompson, M.J., Christensen-Dalsgaard, J., Miesch, M.S., \& Toomre, J., 2003, Ann. Rev. Astron. \& Astrophys., 41, 599

Toomre, J., Zahn, J.-P., Latour, J., \& Spiegel, E.A., 1976, Astrophys. J., 207, 545

Toomre, J., Gough, D.O., \& Spiegel, E.A., 1977, J. Fluid Mech., 79, 1

Toomre, J., Gough, D.O., \& Spiegel, E.A., 1982, J. Fluid Mech., 125, 99

Vasil, G.M., Lecoanet, D., Brown, B.P., Wood, T.S., \& Zweibel, E.G., 2013, Astrophys. J., 773,169

Vauclair, G., Vauclair, S. \& Michaud, G., 1978, Astrophys. J., 223, 920

Zahn, J.P., 1999 ASP Conf. Ser., 173, 121

Zahn, J.-P., Toomre, J., \& Latour, J., 1982, Geophys. Astrophys. Fluid Dynam., 22, 159

Zahn, J.-P., Toomre, J., Spiegel, E.A., \& Gough, D.O., 1974, J. Fluid Mech., 64, 319

Zhao, J., Bogart, R.S., Kosovichev, A.G., Duvall, T.L., Jr., \& Hartlep, T., 2013, Astrophys. J., 774, L29 